\title{
SENSIBILIDAD ANTIMICROBIANA DE LA MICROBIOTA AMBIENTAL DE LAS UNIDADES DE CUIDADOS INTENSIVOS DE UN HOSPITAL PERUANO
}

\author{
José Díaz-Tello ${ }^{1, a}$, Jesús Rojas-Jaimes ${ }^{2, b}$, Jimmy Ibarra-Trujillo ${ }^{3, b}$, Delza Tárraga-Gonzales ${ }^{1, b}$
}

\begin{abstract}
RESUMEN
Con el objetivo de detectar y determinar la sensibilidad antimicrobiana de bacilos Gram negativos y cocos Gram positivos aislados de la microbiota ambiental de los servicios de la Unidad de Cuidados Intensivos (UCl) de Neonatología, de Pediatría y de la Unidad de trasplantes (renal, hepático y general) en un hospital de Lima; se tomaron 80 muestras de superficies inanimadas usando hisopado húmedo. Se identificaron 61 cepas bacterianas que correspondieron a Staphylococcus epidermidis (46,0\%), Alcaligenes sp. (21,3\%), Pseudomonas aeruginosa (16,4\%), Acinetobacter sp. (13,1\%) Staphylococcus aureus (1,6\%) y Staphylococcus haemolyticcus (1,6\%). Acinetobacter y Pseudomonas aeruginosa mostraron una elevada sensibilidad a los antibióticos evaluados, en contraste Alcaligenes sp. y Staphylococcus epidermidis presentaron la mayor resistencia antimicrobiana. Staphylococcus epidermidis y Alcaligenes $s p$. fueron las bacterias que presentaron mayor resistencia a los antibióticos y las que mayormente se aislaron. Se recomienda recurrir a métodos de asepsia y monitoreo sostenidos en las $\mathrm{UCl}$.
\end{abstract}

Palabras clave: Unidades de Cuidados Intensivos; Pruebas de Sensibilidad Microbiana; Microbiota (fuente: DeCS BIREME)

\section{ANTIMICROBIAL SENSITIVITY OF THE ENVIRONMENTAL MICROBIOTA IN THE INTENSIVE CARE UNITS OF A PERUVIAN HOSPITAL}

\begin{abstract}
The objective was to detect Gram-negative bacilli and Gram-positive cocci isolated from the environmental microbiota of the Intensive Care Unit (ICU) departments of Neonatology, Pediatrics, and Transplants (kidney, liver, and general) in a Lima hospital and determine their antimicrobial sensitivity. Eighty samples were obtained from inanimate surfaces using a wet swab. A total of 61 bacterial strains were identified, including Staphylococcus epidermis (46.0\%), Alcaligenes sp. (21.3\%), Pseudomonas aeruginosa (16.4\%), Acinetobacter sp. (13.1\%), Staphylococcus aureus (1.6\%), and Staphylococcus haemolyticus (1.6\%). Acinetobacter sp. and $P$. aeruginosa showed a heightened sensitivity to the antibiotics assessed, while Alcaligenes $s p$. and $S$. epidermidis presented the highest antimicrobial resistance. It is recommended that sustained asepsis and monitoring methods be used in ICUs.
\end{abstract}

Keywords: Intensive Care Units; Microbial Sensitivity Tests; Microbiota (source: MeSH NLM).

\section{INTRODUCCIÓN}

La resistencia de las bacterias patógenas $u$ oportunistas a los antibióticos es un fenómeno progresivo que aparece luego de la introducción de los diferentes antimicrobianos, se desarrolla y se comporta en forma acumulativa en diferentes especies, tiende a la multirresistencia y es detectable tanto en los hospitales como en la comunidad, sobre todo en las últimas décadas ${ }^{(1)}$.
Talbot et al. publicaron una lista de bacterias resistentes a la mayoría de antibióticos disponibles. Estos microorganismos multirresistentes son el Staphylococcus áureus resistente a oxacilina, Escherichia coli, Klebsiella spp., Acinetobacter baumanni, Enterococcus faecium y Pseudomonas aeruginosa ${ }^{(2)}$. En una nota de prensa de la Organización Mundial de la Salud (OMS) en abril de 2015, se señala que la resistencia a los antimicrobianos es una amenaza creciente para la salud pública mundial y que requiere

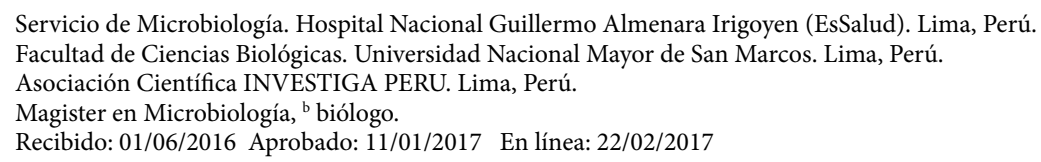

Citar como: Díaz-Tello J, Rojas-Jaimes J, Ibarra-Trujillo J, Tárraga-Gonzales D. Sensibilidad antimicrobiana de la microbiota ambiental de las unidades de cuidados intensivos de un hospital peruano. Rev Peru Med Exp Salud Publica. 2017;34(1):93-7.doi:10.17843/rpmesp.2017.341.2709 
medidas de control inmediatas por parte de la sociedad y de todos los sectores gubernamentales involucrados ${ }^{(3)}$.

En todas las regiones del mundo, una elevada proporción de bacterias responsables de infecciones frecuentes son capaces de resistir la acción de los antibióticos. Más aun al tratarse de infecciones intrahospitalarias (IIH), que son causadas por bacterias muy resistentes como el Staphylococcus aureus resistente a la meticilina (SARM) o bacterias Gram negativas multirresistentes, como Escherichia coli y Klebsiella sp. ${ }^{(4-6)}$.

Se ha reportado que en los países en desarrollo cada día 1,4 millones de pacientes adquieren una IIH ${ }^{(7)}$. El Centro de Control para Enfermedades (CDC) estima que casi dos millones de pacientes adquirirán una infección intrahospitalaria cada año, mientras permanecen en un hospital de los Estados Unidos de Norteamérica, y casi 90 000 de ellos morirán debido a una de estas infecciones, por tanto, es importante involucrar al personal de salud en la prevención y diagnóstico de las $\mathrm{IIH}$, con el fin de reducir la morbimortalidad por estas causas ${ }^{(7,8)}$.

La probabilidad de adquirir una IIH en la Unidad de Cuidados Intensivos (UCI) es alta (40\%), esto debido a factores terapéuticos con efectos de inmunosupresión, a la enfermedad base, a la virulencia y resistencia de los agentes etiológicos circulantes en la unidad hospitalaria, esto conlleva a que cada esquema terapéutico deba ser tratado en forma particular para cada caso ${ }^{(9)}$. Las IIH que ocasionan los microorganismos resistentes tienen un gran impacto sobre los enfermos, pues prolongan sus tratamientos, su tiempo de hospitalización e incrementan su mortalidad. La resistencia también promueve el uso de drogas más tóxicas y más caras ${ }^{(10)}$.

En Perú, Shimabuku et al. determinaron los microorganismos causantes de infecciones en recién nacidos hospitalizados, encontrando que Staphylococcus epidermidis (38,3\%), Staphylococcus aureus $(12,0 \%)$, Klebsiella sp. $(10,0 \%)$ y Alcaligenes fecalis $(4,6 \%)$ fueron los más frecuentes ${ }^{(11)}$.

El objetivo del presente trabajo fue determinar la sensibilidad antimicrobiana de bacilos Gram negativos y cocos Gram positivos aislados en la microbiota ambiental de las UCl de Pediatría, de Neonatología y de las unidades de trasplantes del Hospital Nacional Guillermo Almenara Irigoyen.

\section{EL ESTUDIO}

Se realizó un estudio transversal en la $\mathrm{UCl}$ de Pediatría, de Neonatología y en las de unidades de trasplante renal, hepático y general del Hospital Nacional Guillermo Almenara Irigoyen, durante los meses de marzo a junio del año 2015.
El Hospital Nacional Guillermo Almenara Irigoyen es un hospital de referencia nacional, clasificado como un instituto de salud especializado con un nivel de complejidad III-2, que pertenece al seguro de salud peruano (EsSalud) y está ubicado en el distrito de La Victoria en Lima, Perú.

Se consideró realizar el estudio en las $\mathrm{UCI}$, por ser ambientes cerrados con tránsito restringido, con altos estándares de bioseguridad, a pesar de esto, estas áreas presentan factores de riesgo para adquirir una infección intrahospitalaria como la inmunosupresión y/o inmunodeficiencia, en este caso, en pacientes trasplantados, pediátricos y neonatos.

La frecuencia mensual de pacientes internados en la UCl de Pediatría y de Neonatología es de 6 y 16 respectivamente. Las $\mathrm{UCl}$ de trasplantes (hepático, renal y general) presentan una frecuencia mensual de 7,8 y 15 pacientes, respectivamente.

Los materiales considerados para la toma de muestra se encuentren en contacto directo con los pacientes, como la baranda de la cama, el bacín (chata), la manija de la puerta del baño, la pared y el piso; en el caso del personal de salud o del administrativo, los materiales y equipos escogidos que manipulan con frecuencia fueron las carpetas clínicas, la ventana, la manija principal de la puerta de la UCI, la manija de coche de medicamentos y el monitor del ventilador mecánico.

\section{TOMA DE MUESTRA}

Se realizó con un hisopo estéril húmedo, que se froto sobre el área determinada con una inclinación aproximada de $30^{\circ}$, el frotado se realizó cuatro veces, cada una en dirección opuesta sobre una superficie aproximada de $100 \mathrm{~cm}^{2}(10 \mathrm{~cm} \times 10 \mathrm{~cm})$. Se tomaron 80 muestras (16 de cada $\mathrm{UCl}$ ) todas las muestras fueron etiquetadas correctamente para su posterior traslado al laboratorio, el medio de transporte utilizado fue el de Amies, medio enriquecido con iones fosfato, calcio y magnesio que actúan como solución tampón y ayuda a conservar la permeabilidad de la membrana bacteriana.

\section{AISLAMIENTO E IDENTIFICACIÓN BIOQUÍMICA}

En el Laboratorio de Microbiología se sembraron las muestras en medios de cultivos comunes y selectivos, según el grupo de bacterias; para su identificación se consideraron las recomendaciones descritas en el Manual de Bergey's ${ }^{(12)}$. Además, a todas las cepas aisladas se les realizo la tinción de Gram como criterio taxonómico fundamental para la correcta identificación del microorganismo. 


\section{PRUEBAS DE SENSIBILIDAD A ANTIMICROBIANOS}

Se realizaron antibiogramas de todas las cepas según el método disco difusión de Kirby y Bauer ${ }^{(13)}$ y se evaluó la sensibilidad según los criterios de la Clinical and Laboratory Standards Institute (CLSI) ${ }^{(14) .}$ Los discos de antimicrobianos de uso clínico que se utilizaron fueron: amikacina (Ak) $30 \mu \mathrm{g}$; gentamicina (Ge) $10 \mu \mathrm{g}$; ciprofloxacina (Cip) $5 \mu \mathrm{g}$, ampicilina (A) $10 \mu \mathrm{g}$; ceftazidima (Caz) $30 \mu \mathrm{g}$; cotrimoxazol (Stx) $25 \mu \mathrm{g}$; cefepime (CMP) $30 \mu \mathrm{g}$; imipenem (IMP) 10 $\mu \mathrm{g}$; piperaciclina /tazobactan (TZP) $110 \mu$ g; oxacilina (OXA) $1 \mu \mathrm{g}$; eritromicina (E) $15 \mu \mathrm{g}$; vancomicina (VAN) $30 \mu \mathrm{g}$, y clindamicina (Da) $2 \mu \mathrm{g}$. (Laboratorios EMV de Chile). Para un control de calidad de los discos se evaluaron cepas de Escherichia coli ATCC; 25922, Pseudomonas aeruginosa ATCC: 27853 y Staphylococcus aureus ATCC 25923.

Se procedió al hallazgo de las frecuencias y porcentajes, tanto para la determinación de los microorganismos hallados en las diferentes $\mathrm{UCl}$ estudiadas, como para la cuantificación de la resistencia a los antibióticos.

\section{HALLAZGOS}

\section{AISLAMIENTO E IDENTIFICACIÓN BIOQUÍMICA}

Se aislaron 61 cepas bacterianas en las diferentes $\mathrm{UCl}$ considerados para este estudio. Las bacterias aisladas fueron: Acinetobactersp., Alcaligenes sp., Pseudomonas aeruginosa ( $P$. aeruginosa), Staphylococcus aureus (S. aureus), Staphylococcus epidermidis (S. epidermidis) y
Staphylococcus haemolyticcus (S. haemolyticcus), en el caso de Acinetobacter sp. y Alcaligenes sp. solo se llegó hasta la identificación del género según método de aislamiento clásico. El microorganismo que se encontró con mayor frecuencia fue $S$. epidermidis con $46,0 \%$ de total de cepas aisladas. Las bacterias que se identificaron en menor porcentaje fueron $S$. aureus y $S$. haemolyticcus (ambas con 1,6\%). La mayor cantidad de microorganismos se aislaron en la $\mathrm{UCl}$ de Trasplante de Hígado, Renal y General, en contraste con la UCl de Neonatología donde se encontró la menor cantidad de bacterias (Tabla1).

\section{PRUEBAS DE SENSIBILIDAD A ANTIMICROBIANOS}

Las bacterias Acinetobacter sp. y $P$. aeruginosa presentaron una elevada sensibilidad a los antibióticos evaluados. Además, se pudo observar que la especie Alcaligenes fue la bacteria Gram negativa que presentó un mayor patrón de resistencia antimicrobiana, además, se aisló en la mayoría de las manijas de puerta y carpetas clínicas. Entre los Gram positivos el $S$. aureus y el $S$. epidermidis presentaron un mayor patrón de resistencia a los antibióticos evaluados. $S$. epidermidis fue el que se halló con mayor frecuencia en las carpetas clínicas y monitores de los equipos de respiración asistida. Las cepas que presentaron resistencia a oxacilina fueron $S$. aureus, S. epidermidis y S. haemolyticcus. La oxacilina se utilizó para determinar la resistencia a meticilina (Tabla 2). El antibiótico que presentó mayor resistencia fue la ampicilina y todas las especies evaluadas en el presente estudio fueron sensibles a vancomicina.

Tabla 1. Microorganismos aislados según su distribución en las diferentes UCl del Hospital Nacional Guillermo Almenara Irigoyen. Lima, Perú. 2015

\begin{tabular}{|c|c|c|c|c|c|c|}
\hline Microorganismos & $\begin{array}{l}\text { UCl de } \\
\text { Pediatría }\end{array}$ & $\begin{array}{c}\text { UCI de } \\
\text { Neonatología }\end{array}$ & $\begin{array}{c}\text { UCI de } \\
\text { Trasplante } \\
\text { de Hígado }\end{array}$ & $\begin{array}{l}\text { UCl de } \\
\text { Trasplante } \\
\text { Renal }\end{array}$ & $\begin{array}{c}\text { UCl de } \\
\text { Trasplante } \\
\text { General }\end{array}$ & $\begin{array}{l}\text { Total } \\
\text { N (\%) }\end{array}$ \\
\hline Acinetobacter sp. & - & - & 4 & 1 & 3 & $8(13,1)$ \\
\hline Alcaligenes sp. & - & - & 6 & 4 & 3 & $13(21,3)$ \\
\hline Pseudomonas aeruginosa & - & - & 4 & 3 & 3 & $10(16,4)$ \\
\hline Staphylococcus aureus & - & - & - & - & 1 & $1(1,6)$ \\
\hline Staphylococcus epidermidis & 8 & 7 & - & 7 & 6 & $28(46,0)$ \\
\hline $\begin{array}{l}\text { Staphylococcus } \\
\text { haemolyticcus }\end{array}$ & 1 & - & - & - & - & $1(1,6)$ \\
\hline Total n (\%) & $9(14,6)$ & $7(11,5)$ & $14(23,0)$ & $15(24,6)$ & $16(26,3)$ & $61(100,0)$ \\
\hline
\end{tabular}

UCl: Unidad de Cuidados Intensivos 
Tabla 2. Resistencia a los antibióticos según bacillos Gram negativos y cocos Gram positivos, en las UCl del Hospital Nacional Guillermo Almenara Irigoyen. Lima, Perú. 2015

\begin{tabular}{|c|c|c|c|}
\hline \multirow[b]{2}{*}{ Antibiótico $(\mu \mathrm{g})$} & \multicolumn{3}{|c|}{ Microorganismos gramnegativos } \\
\hline & Acinetobacter $n(\%)$ & Alcaligenes $n(\%)$ & P. auriginosa $n(\%)$ \\
\hline Amikacina $30 \mu \mathrm{g}$ & 0,0 & $1(7,6)$ & 0,0 \\
\hline Gentamicina $10 \mu \mathrm{g}$ & 0,0 & $1(7,6)$ & 0,0 \\
\hline Ciprofloxacina $5 \mu \mathrm{g}$ & $3(37,5)$ & $1(7,6)$ & 0,0 \\
\hline Ceftazidima $30 \mu \mathrm{g}$ & 0,0 & $3(22,8)$ & $3(30,0)$ \\
\hline Cefepime $30 \mu \mathrm{g}$ & 0,0 & $2(15,2)$ & $1(10,0)$ \\
\hline Piperaciclina /Tazobactan $110 \mu \mathrm{g}$ & 0,0 & $2(15,2)$ & 0,0 \\
\hline \multirow[t]{2}{*}{ Imipenem $30 \mu \mathrm{g}$} & 0,0 & $1(7,6)$ & $5(50,0)$ \\
\hline & \multicolumn{3}{|c|}{ Microorganismos grampositivos } \\
\hline Antibiótico $(\mu \mathrm{g})$ & S. epidermidis $n(\%)$ & S. aureus $n(\%)$ & S. haemolyticus $n(\%)$ \\
\hline Ampicilina $30 \mu \mathrm{g}$ & $26(92,8)$ & $1(100,0)$ & $1(100,0)$ \\
\hline Ciprofloxacina $5 \mu \mathrm{g}$ & $8(28,5)$ & 0,0 & $1(100,0)$ \\
\hline Clindamicina $2 \mu \mathrm{g}$ & $11(39,2)$ & 0,0 & $1(100,0)$ \\
\hline Eritromicina $15 \mu \mathrm{g}$ & $14(50,0)$ & 0,0 & $1(100,0)$ \\
\hline Cotrimoxazol $25 \mu \mathrm{g}$ & $7(25,0)$ & 0,0 & $1(100,0)$ \\
\hline Oxacilina $5 \mu \mathrm{g}$ & $12(42,8)$ & $1(100,0)$ & $1(100,0)$ \\
\hline Vancomicina $30 \mu \mathrm{g}$ & 0,0 & 0,0 & 0,0 \\
\hline
\end{tabular}

\section{DISCUSIÓN}

La especie bacteriana aislada con mayor frecuencia de las carpetas clínicas y monitores fue $S$. epidermidis, además de presentar la mayor resistencia entre los cocos Gram positivos. Esta bacteria fue considerada por mucho tiempo como un microorganismo contaminante de cultivos, sin embargo, ahora se le reconoce como un patógeno importante ${ }^{(15)}$. Entre los Gram negativos la bacteria aislada con mayor frecuencia en manijas de puertas y carpetas clínicas fue Alcaligenes sp. con un alto patrón de resistencia. Esta bacteria podría convertirse en un patógeno oportunista en personas inmunosuprimidas ${ }^{(16)}$. Este hallazgo supondría que el contacto con las manijas y carpetas clínicas podrían ser factores de riesgo para la adquisición de $\mathrm{IIH}$.

$P$. aeruginosa es un agente importante de $\mathrm{IlH}$, mayormente multirresistente, aunque en nuestro estudio mostro una buena sensibilidad a los antibióticos (17). Wang et al. demostraron, en un estudio similar, que $P$. aeruginosa tenía una alta frecuencia en los grifos de agua ubicados en las UCI de los hospitales ${ }^{(16)}$.

Acinetobacter sp. reportó ser sensible a la mayoría de antibióticos, este microorganismo es conocido por ser un oportunista de importancia en el ambiente hospitalario ${ }^{(18)}$. Es probable que los aislamientos de $P$. aeruginosa y Acinetobacter $\mathrm{sp}$. de nuestro estudio mostrasen un patrón sensible a los antibióticos por provenir de fuentes de agua (humificadores) y de personas sin previa exposición a las drogas, comportándose como cepas silvestres.
Las $\mathrm{UCl}$ de las unidades de trasplantes presentaron la mayor cantidad de aislamientos, en contraste con la $\mathrm{UCl}$ de Neonatología que reporto un solo tipo de aislamiento ( $S$. epidermidis), una explicación válida seria que las unidades de trasplantes alojan pacientes en su mayoría adultos (con una microbiota mayor y variada) en comparación con los pacientes de las UCI de Pediatría y de Neonatología. Otra posible explicación sería que la capacidad de movilizarse en los pacientes de las $\mathrm{UCl}$ de trasplantes propiciaría la contaminación al contacto con áreas cercanas por fluidos corporales, a diferencia de las $\mathrm{UCl}$ de Pediatría y Neonatología donde los pacientes están mayormente postrados con mayor restricción en el acceso.

S. epidermidis fue la bacteria de se aisló con mayor frecuencia en nuestro estudio, este hallazgo es similar a lo reportado por Paz-Rojas et al, que tomó muestras de secreción de heridas, de exudados traqueales, de aspirados bronquiales y de catéteres de pacientes de diferentes ambientes de un centro hospitalario español, en cuyas $\mathrm{UCl}$ se demostró que $S$. epidermidis fue el estafilococo coagulasa negativo más frecuente y de mayor relevancia asociada a infecciones intrahospitalarias ${ }^{(19)}$.

Con respecto a las pruebas de sensibilidad antimicrobiana, se muestra que Acinetobacter sp. y $P$. aeruginosa presentaron una elevada sensibilidad a los antibióticos evaluados, aunque la Pseudomonas aeruginosa presentó una elevada resistencia al imipenem. A diferencia del presente estudio, se considera generalmente a Acinetobacter sp. y Pseudomonas aeruginosa como bacterias multirresistentes aisladas en la $\mathrm{UCI}^{(18,20-22)}$.

Como limitación podemos mencionar que los aislamientos fueron realizados en superficies inanimadas 
y no directamente de pacientes, sin embargo, es sabido que existe una relación directa entre la contaminación de superficies inanimadas y la contaminación de los pacientes ${ }^{(18)}$. A pesar de ser solo tres las $\mathrm{UCl}$ estudiadas, consideramos que el estudio proporciona un acercamiento al problema de la resistencia bacteriana en áreas críticas en hospitales de Perú.

En conclusión, las bacterias aisladas con mayor frecuencia y resistencia a los antibióticos en superficies inanimadas de las UCl del Hospital Nacional Guillermo Almenara Irigoyen fueron $S$. epidermidis y Alcaligenes $\mathrm{sp}$. Se recomienda mejorar los protocolos de asepsia en las personas y ambientes en las $\mathrm{UCl}$ hospitalarias, realizar una vigilancia epidemiológica de la microbiota en dichas unidades, con el fin de evitar casos de morbimortalidad en los pacientes y realizar estudios para evaluar la relación entre la microbiota hospitalaria de las $\mathrm{UCl}$ y los aislamientos de pacientes febriles.

\section{REFERENCIAS BIBLIOGRÁFICAS}

1. Fica A. Resistencia antibiótica en bacilos Gram negativos, cocáceas Gram positivas y anaerobios. Implicancias terapéuticas. Rev Med Clin Condes. 2004;25(3):432-44.

2. Talbot GH, Bradley J, Edwards J. Bad bugs no drugs: an update on the development pipeline from the antimicrobial availability task force of the Infectious disease Society of America. Clin Inf Dis. 2006; 42(5):657-68.

3. Organización Mundial de la Salud. Resistencia a los antimicrobianos [Internet]. Ginebra: Centro de Prensa. Disponible en: http://www.who.int/ mediacentre/factsheets/fs194/es/.

4. Alvares C, Cortes J, Arango A, Corre C, Leal A, Grebo. Resistencia antimicrobiana en Unidades de Cuidados Intensivos de Bogotá, 2001 - 2003. Rev Salud Publica (Bogota). 2006;8 Suppl 1:86-101.

5. Hechavarría Soulary JC, Suárez Domínguez R, Armaignac Ferrer G, del Pozo Hessing C. Infección nosocomial en la Unidad de Cuidados Intensivos. MEDISAN. 2001; 5(3):12-17.

6. Basulto BarrosoI MM, Galdós Sánchez MDC, Carr González J, Díaz Agüero $H$. Infección nosocomial respiratoria en la Unidad de Cuidados Intensivos. Revista Archivo Médico de Camagüey. 2008;13(2):1-10.

7. Garro Nuñez GM, Quispe Pardo ZE. Protocolo: Estudio prevalencia de infecciones Intrahospitalarias [Internet]. Lima: Ministerio de Salud/ Dirección General de Epidemiologia; 2014 [citado el 24 de mayo del 2016]. Disponible en: http://www.minsa.gob. $\mathrm{pe/dgsp/observatorio/documentos/}$ infecciones/Protocolo\%20Estudio\%20 de\%20Prevalencia_DGE.pdf

8. Unahualekhaka A. Epidemiología de las infecciones asociadas a la atención de salud. United Kingdom: International Federation of Infection Control;
2015. [citado el 15 de mayo del 2016]. Disponible en: http://theific.org/wpcontent/uploads/2014/08/Spanish_ ch3_PRESS.pdf

9. Zaragoza R, Ramírez P, López-Pueyo M. [Nosocomial infections in intensive care units]. Enferm Infecc Microbiol Clin. 2014;32(5):320-7. [Article in Spanish].

10. World Health Organization, Department of Communicable Disease, Surveillance and Response. Prevention of Hospital-acquired Infections. Geneva, Switzerland: World Health Organization; 2002. Publication WHO/CDS/CSR/ $\mathrm{EPH} / 2002.12$.

11. Shimabuku R,Velásquez P,Yábar J,Zerpa R,Arribasplata G,Fernández, et al. Etiología y susceptibilidad antimicrobiana de las infecciones neonatales. An Fac med. 2004;66(1):19-24.

12. Garrity GM, Bell JA, Lilburn TG. Taxonomic outline of the procaryotes. Bergey's manual of systematic bacteriology. New York: SpringerVerlag; 2004.

13. Bauer A, Kirby W, Sherris J, Turck M. Antibiotic susceptibility testing by a standardized sing disk method. Am J Clin Pathol. 1966; 45(4):493-6.

14. CLSI. Performance Standards for Antimicrobial Susceptibility Testing. 25th ed. CLSI supplement M100. Wayne: Clinical and Laboratory Standards Institute; 2015.

15. Mandel G, Bennett JE, Dolin R. Principles and Practices of Infectious Diseases. 5th ed. Philadelphia: Churchill Livingstone; 2000.

16. Wang JL, Chen ML, Lin YE, Chang SC, Chen YC. Association between contaminated faucets and colonization or infection by nonfermenting gram-negative bacteria in intensive care units in Taiwan. J Clin Microbiol. 2009;47(10):3226-30. doi: 10.1128/JCM.00034-09.
17. Gómez,J,Alcántara M,Simarro E,Martínez B, Ruiz J, Guerra M, et al. Bacteriemias por Pseudomonas aeruginosa: epidemiología, clínica y tratamiento. Estudio prospectivo de siete años. Rev Esp Quimioterap. 2002;15(4):360-5.

18. Paz Rojas E, Ponce de León Pandolfi D, Ramirez Ponce R. Resistencia bacteriana en cuidados intensivos y tendencia actual: Departamento de Cuidados Críticos, Servicio de cuidados intensivos del Hospital Guillermo Almenara Irigoyen, Essalud, Lima Perú 2004-2006. Acta med peruana. 2008;25(3):140-7.

19. Lozano C, Aspiroz C, Gómez-Sanz E, Tirado G, Fortuño B, Zarazaga $\mathrm{M}$, et al. Caracterización de cepas de Staphylococcus epidermidis y $S$. haemolyticus resistentes a meticilina y linezolid en un hospital español. Enferm Infecc Microbiol Clin. 2013;31(3):13641. doi: 10.1016/j.eimc.2012.08.006.

20. Sales VM, Oliveira E, Célia R, Ramos Gonçalves F, Carvalho de Melo C. Análise microbiológica de superfícies inanimadas de uma Unidade de Terapia Intensiva e a segurança do paciente. Rev Enf Ref. 2014;4(3):45-53. doi: 10.12707/RIII1293.

21. Lemos EV, De la Hoz Restrepo F, Alvis N, Quevedo E, Cañon O, León Y. Mortalidad por Acinetobacter baumannii en unidades de cuidados intensivos en Colombia. Rev Panam Salud Publica. 2011;30(4):287-94.

22. Miranda MC, Pérez F, Zuluaga T, Olivera R, Correa A, Reyes SL, et al. Resistencia a antimicrobianos de bacilos Gramnegativos aislados en unidades de cuidado intensivo en hospitales de Colombia, WHONET 2003, 2004 y 2005. Biomédica. 2006;26(3):424-33.

Autor Corresponsal: Jesús Rojas Jaimes

Dirección: Km 19, Villa, Panamericana Sur, Villa El Salvador

Teléfono: (511) 6106400

Correo electrónico:jesus.rojas.jaimes@gmail.com 